\title{
Suspect the unsuspected: an unusual case of primary cutaneous mucormycosis at a peripheral intravenous catheter insertion site. a case report and literature review
}

\begin{abstract}
Fungal Infections can have atypical presentation. This makes the diagnosis of fungal infections very challenging and in many cases increases morbidity and in unfortunate cases causes' mortality. Mucormycosis is a rare fungus which can present with wide clinical spectrum and can mislead as other etiologies. This fungus manifests itself mostly in immune compromised hosts. High index of suspicion is needed for accurate diagnosis. Prompt treatment includes combination of surgical debridement and antifungals. Early diagnosis and initiation of appropriate management can significantly decrease morbidity and mortality. Histological diagnosis is the only means of confirming mucormycosis infection. Our case is a typical example of atypical presentation of mucormycosis in an immune compromised patient which resulted in limb amputation.
\end{abstract}

Keywords: mucormycosis, ubiquitous saprophytic organisms, zygomycetes, thrombophlebitis, amphotericin $b$
Volume 4 Issue 6 - 2017

\author{
Sri Lakshmi Yeruva,' 'Vijay Kodadhala,' Martin \\ Ucanda, ${ }^{2}$ Ankit Mahajan,' BabakShokrani, ${ }^{3}$ \\ JhansiGajjala ${ }^{2}$ \\ 'Department of Internal Medicine, Howard University Hospital, \\ USA \\ ${ }^{2}$ Department of Infectious Diseases, Howard University \\ Hospital, USA \\ ${ }^{3}$ Department of Pathology, Howard University Hospital, USA
}

Correspondence: Sri Lakshmi Yeruva, Department of Internal Medicine, Howard University Hospital,Washington DC, USA, Email dryeruva24@gmail.com

Received: May 18,2017 | Published: June 26, 2017

\section{Introduction}

Mucormycosis is a rare infection caused by hyaline filamentous fungi which are ubiquitous saprophytic organisms. They belong to class zygomycetes and order mucorales. ${ }^{1,2}$ Usually they grow rapidly and produce wide hyaline, aseptate or poorly septate ribbon-like hyphae in tissues. ${ }^{3}$ This infection is characterized by angio-invasion resulting in soft tissue destructionby thrombosis and necrosis. ${ }^{4}$ They cause potentially fatal infection in humans, more commonly seen in immune compromised individuals. Although mucormycosis can involve any part of the body; primary cutaneous form has been particularly rare.

\section{Case report}

A 54 yr old Hispanic male with medical history of Ulcerative colitis for 7years presented with left sided abdominal pain, nausea, vomiting, bloody diarrhea and low grade fever $(100.30 \mathrm{~F})$ for 2day duration. His home medication included prednisone $20 \mathrm{mg}$ daily for the past 2 years and mesalamine. He denied smoking, alcohol or illicit drug use and family history was unremarkable. He is allergic to penicillin, administration of which causes moderate generalized itching and hives. Physical examination was normal except for mild left lower quadrant tenderness. Initial laboratory data was unremarkable except for leucocytosis with a WBC count of $15,800 / \mathrm{mm}^{3}$ and creatinine of $2 \mathrm{mg} / \mathrm{dl}$. He was admitted with the diagnosis of exacerbation of ulcerative colitis and was started on intravenous metronidazole, ciprofloxacin and steroids. Patient was placed on insulin for steroid induced hyperglycemia.

Three days into hospital admission and initiation of above mentioned treatment, physical examination of right hand revealed a tender, erythematous in duration around the insertion site of peripheral venous catheter. Peripheral catheter was removed. Bacterial blood cultures x 2 were ordered and patient was started on empiric treatment with Vancomycin and Aztreonam for presumed superficial thrombophlebitis. At that time fungal blood cultures were not requested. By day 5 of hospital stay, despite change of antibiotics, his right hand continued to become progressively swollen and eventually developed into a tender erythematous lesion $(15 \times 7 \mathrm{~cm})$ with blisters. Bacterial blood cultures did not grow any organisms.

Onday 6, patient complained of decreased of sensation to pain and touch on his right hand below the elbow. Physical examination of the same hand revealed loss of sensation to fine touch and decreased sensation to pain, crude touch and temperature from mid forearm to fingers. Vascular exam revealed absent pulses of right radial and ulnar arteries. Based on the physical examination findings critical limb ischemia secondary to compartment syndrome was highly suspected and vascular surgery team was consulted. Patient received exploration of right brachial, radial and ulnar artery procedure. Intraoperatively, multiple intraluminal clots were removed from both radial and ulnar arteries. Thrombus was sent for histo-pathological studies. Intraoperative arteriogram showed no flow beyond mid forearm. Patient was offered above elbow amputation of the right upper limb for non salvageable ischemic limb.

Histopathological examination with Gram stain (Figure 1) and Methenaminesilver stain (Figure $2 \& 3$ ) of thrombus revealed wide, non septate hyphae with right angle branching. Tissue biopsy showed necrosis, vasculitis, thrombosis and fungal vascular invasion suggesting mucormycosis. Amphotericin B was started and patient was transferred to another facility upon his request for limb amputation. 


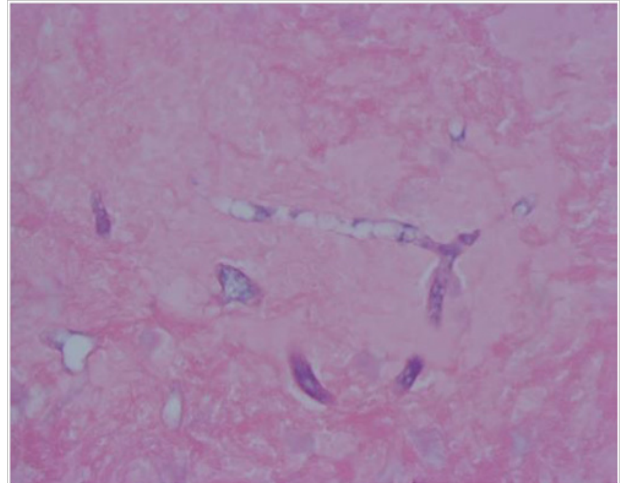

Figure I PAS.

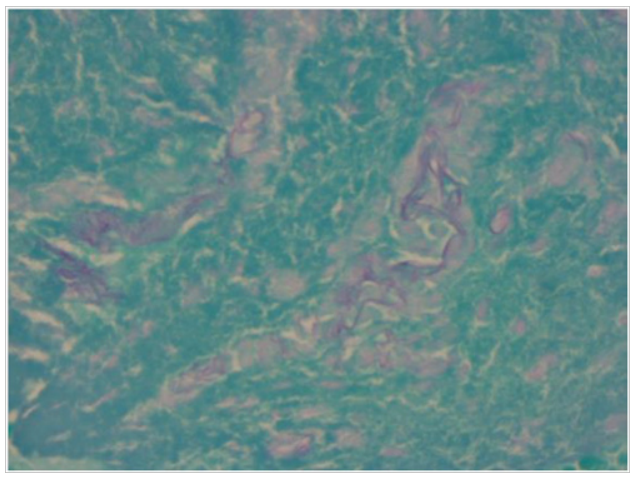

Figure 2 Gomorimethenamine silver stain.

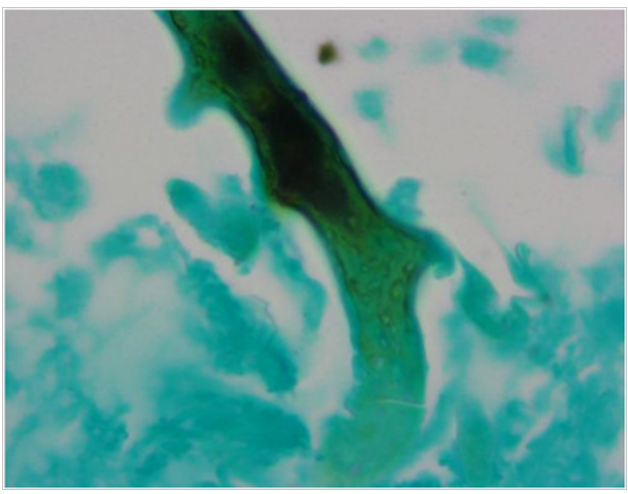

Figure 3 Gomorimethenamine silver stain.

\section{Discussion}

Mucormycosis is a life threatening infection commonly seen in patients with acquired or pharmacological immunosuppression with mortality rate as high as $50-60 \%{ }^{5}$ Six clinical forms have been described which include rhinocerebral, cutaneous, pulmonary, gastrointestinal, disseminated and renal. The major risk factors for mucormycosis include uncontrolled diabetes mellitus in ketoacidosis, other forms of metabolic acidosis, treatment with corticosteroids, organ or bone marrow transplantation, neutropenia, trauma and burns, malignant hematologic disorders, and deferoxamine therapy in patients receiving hemodialysis. ${ }^{6}$

Our patient had multiple risk factors for Mucormycosis namely Ulcerative Colitis, immunosuppressive agents and renal impairment. As erythematous in duration was at the peripheral venous catheter insertion site, we treated with antibiotic therapy empirically for possible superficial thrombophlebitis. Unfortunately patient's clinical condition got worse leading to surgical exploration of affected arm and histological studies of debrided tissue revealed the diagnosis of Mucormycosis.

The prevalence of mucormycosis is increasing; with growing numbers of highly immunocompromised individuals. ${ }^{7}$ Cutaneous mucormycosis usually results from inoculation of the spores into the dermis. Innoculum could entre site from disruption of local defense mechanisms like burns, trauma, surgery, arterial lines, insulin injection sites, biopsy site, contaminated splints, dressings, tattoos and insect bites. ${ }^{8-14}$

Clinical presentation of cutaneous mucormycosis varies with the extent of injury and immune status of the patient. In an immunecompetent host, the onset is sub-acute with vesicles or pustules that may eventually progress into eschars. On the contrary, the immunecompromised host usually presents with a rapidly progressive gangrene like form with centrally necrotic lesions surrounded by erythematous, ecchymotic or cellulitic looking skin. ${ }^{15}$ As our patient was immune-compromised, she had similar clinical presentation. Although cutaneous mucormycosis is associated with lower mortality rate compared to other clinical types, it has high long term morbidity if not diagnosed early and treated appropriately. Per one analysis, mortality was $4-10 \%$ for localized infection, $26-43 \%$ for cutaneous zygomycosis with deep extension, and $50-94 \%$ for disseminated disease. ${ }^{15}$

Definitive diagnosis of cutaneous mucormycosis is based on a tissue biopsy of the involved site, which classically shows non septate or sparsely septate, broad (6-25mm), irregular hyphae with right angle branching. Appropriate management includes early diagnosis and treatment with antifungals and surgical debridement of the involved tissue. Adequately controlling the modifiable factors leading to immune compromised state for example treating diabetic ketoacidosis is also important part of management of Mucormycosis infection. One study revealed that delay in initiation of antifungal treatment resulted in increase in mortality by two folds. ${ }^{16}$

Amphotericin B is the drug of choice. Lipid formulation of this drug is preferred as it is associated with less nephrotoxicity. Patients are needed to be treated for several weeks with amphotericin B to see the clinical response. Once significant clinical response is seen, then amphotericin B can be switched with oral azole drugs like posaconazole or isavuconazole. Therapy should continue until there is clinical resolution of the signs and symptoms of infection, as well as resolution of radiographic signs of active disease; therapy should also continue until reversal of underlying immunosuppression has been achieved, when feasible.

\section{Conclusion}

As peripheral venous catheterization is a very common procedure, high index of clinical suspicion is warranted for early diagnosis, especially in immune-compromised patients and those not responding to the standard therapy. Delay in diagnosis can lead to organ dysfunction either single or multiple and in unfortunate cases demise. Given the high rates of morbidity mortality, appropriate therapy with early surgical debridement and antifungal therapy should be promptly instituted for improved survival.

\section{Authors contribution}

Sri Lakshmi Yeruva, Vijay Kodadhala, Martin Ucanda and Ankit Mahajan contributed extensive review of literature and paper drafting; 
Babak Shokrani confirmed the tissue diagnosis and Jhansi Gajjala primarily managed the patient and overviewed the paper.

\section{Acknowledgements}

None.

\section{Conflict of interest}

The author declares no conflict of interest.

\section{References}

1. Adam RD, Hunter G, DiTomasso J, et al. Mucormycosis: emerging prominence of cutaneous infections. Clin Infect Dis. 1994;19(1):67-76.

2. Howard DH. Classification of the mucorales: Moderator mucormycosis Ann Intern Med. 1980;93:93-94.

3. Rippon JW. Medical mycology: The pathogenic fungi and the pathogenic actinomycetes. 3rd ed. USA: WB Saunders; 1988. p. 681-713.

4. Spellberg B, Edwards J, Ibrahim A. Novel perspectives on mucormycosis pathophysiology, presentation and management. Clin Microbiol Rev. 2005;18(3):556-569.

5. Tapia EO, Chahín AC, Concha FC. Primary cutaneous mucormycosis: two case reports and literature. Rev Chil Infect. 2011;28(3):269-273.

6. Sugar AM. Agents of mucormycosis and related species. In: Mandell GL, Bennett JE, editors. Principles and practice of infectious diseases. 6 th ed. USA: Elsevier Churchill Livingstone; 2005.

7. Skiada A, Petrikkos G. Cutaneous zygomycosis. Clin Microbiol Infect. 2009; 15(Suppl 5):41-45.
8. Bethge WA, Schmalzing M, Stuhler G. Mucormycoses in patients with haematologic malignancies: an emerging fungal infection. Haematologica. 2005;90(6):e62-e64.

9. Hampson FG, Ridgway EJ, Feeley K, et al. A fatal case of disseminated zygomycosis associated with the use of blood glucose self-monitoring equipment. $J$ Infect. 2005;51(5):e269-e272.

10. Kapadia S, Polenakovik H. Cutaneous zygomycosis following attempted radial artery cannulation. Skinmed. 2004;3(6):336-338.

11. Baraia J, Muñoz P, Bernaldo de Quirós JCL, et al. Cutaneous Mucormycosis in a Heart Transplant Patient Associated with a Peripheral Catheter. Eur J Clin Microbiol Infect Dis. 1995;14(9):813-815.

12. Chander J, Kaur J, Attri A, et al. Primary cutaneous zygomycosis from a tertiary care centre in north-west India. Indian J Med Res. 2010;131:765770

13. Lineberry KD, Boettcher AK, Blount AL, et al. Cutaneous mucormycosis of the upper extremity in an immunocompetent host: case report. J Hand Surg Am. 2012;37(4):787-791.

14. Cindy JC, Melissa RM, Maxwell AF, et al. Primary cutaneous mucormycosis at sites of insulin injection. Journal of American Academy of Dermatology. 2011;64(5):e79-e81.

15. Skiada A, Rigopoulos D, Larios G, et al. Global epidemiology of cutaneous zygomycosis. Clin Dermatol. 2012;30(6):628-632.

16. Chamilos G, Lewis RE, Kontoyiannis DP. Delaying amphotericin Bbased frontline therapy significantly increases mortality among patients with hematologic malignancy who have zygomycosis. Clin Infect Dis. 2008;47(4):503-509. 